مسعود نادرى ورندى'، على كيا لاشكى'، رامين ويسى ' و على شيخ الاسلامى

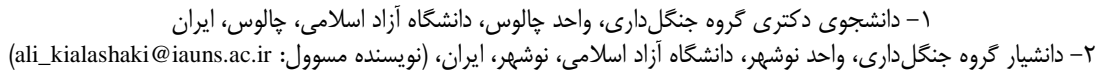

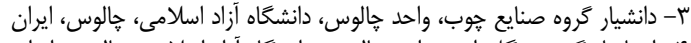

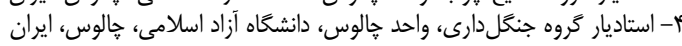

تاريخ يذيرش:TV/T/T)

تاريخ دريافت:

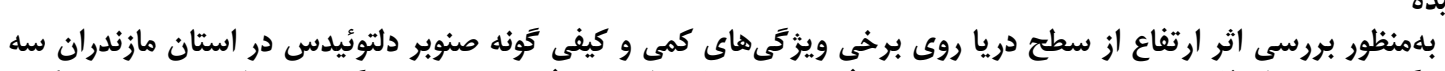

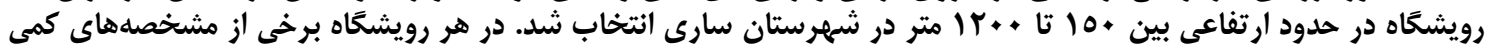

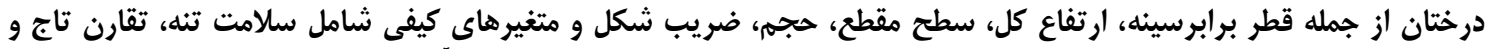

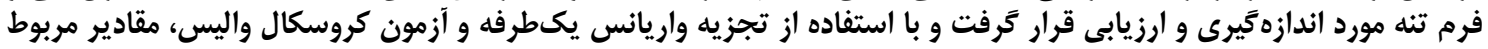

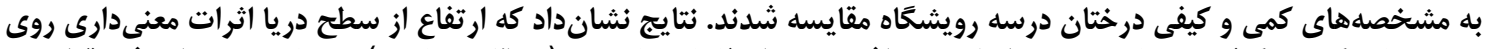

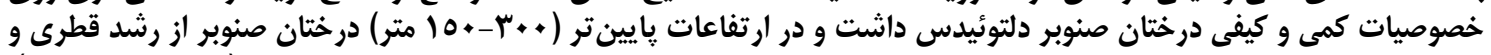

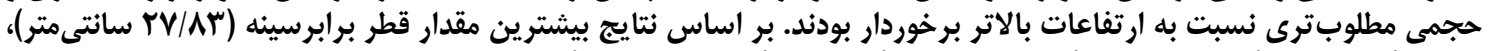

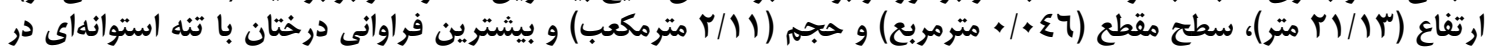

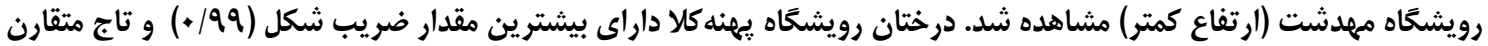

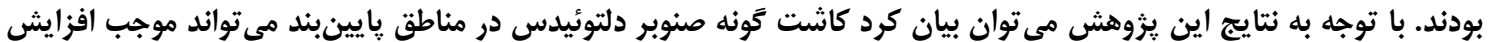

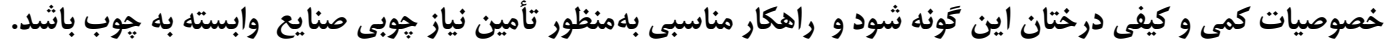

وازههاى كليدى: جنغَل كارى، ويزَّى هاى جنَّلشناسى، رشد قطرى و حجمى، ار تفاع از سطح دريا آزاد

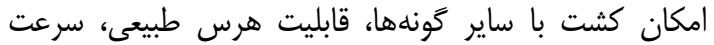

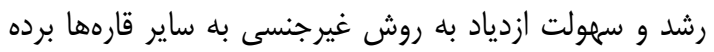

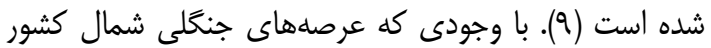

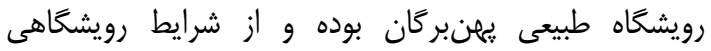

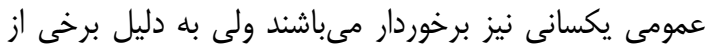

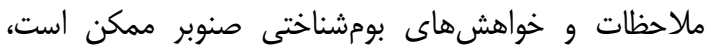

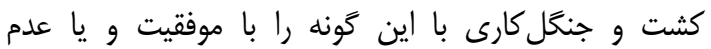

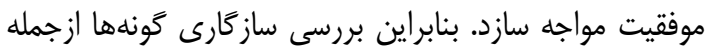

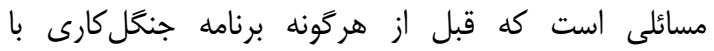

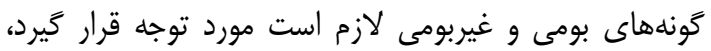

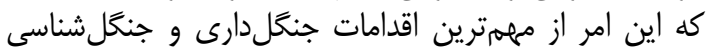

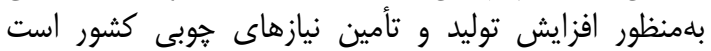

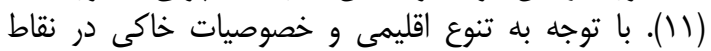

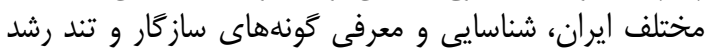

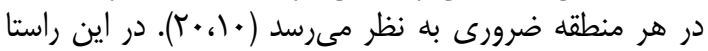

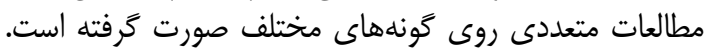

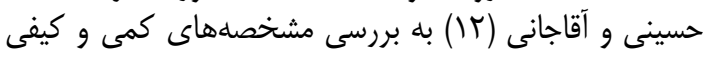

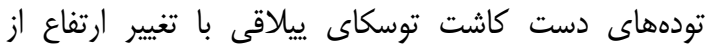

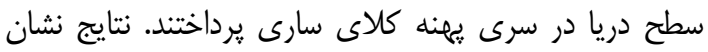

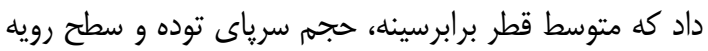

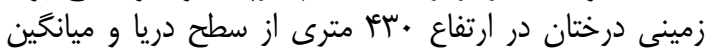

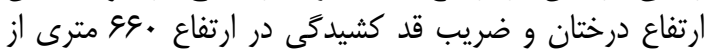

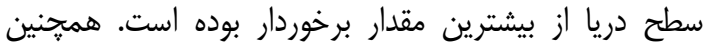

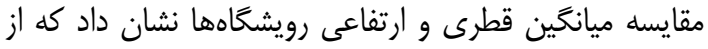

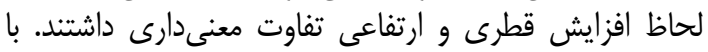

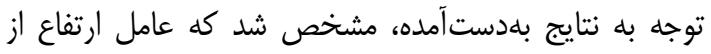

طعى ساليان اخير، افزايش جمعيت در كشور، نياز روزافزون

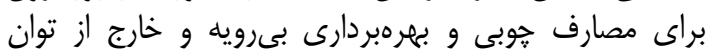

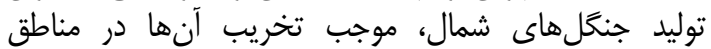

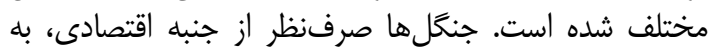

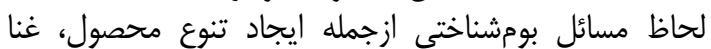

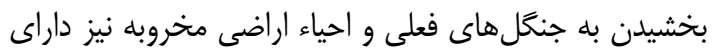

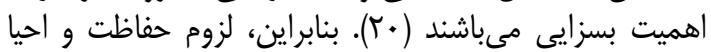

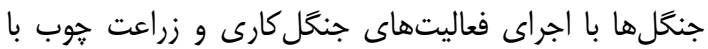

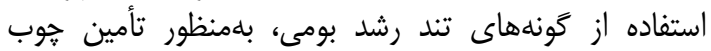

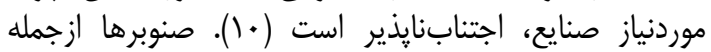

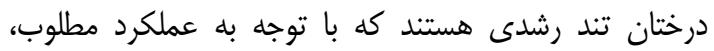

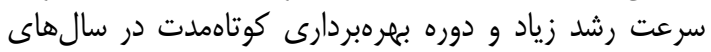

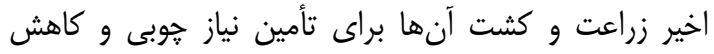

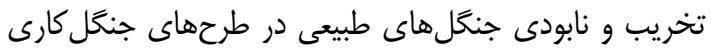

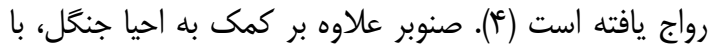

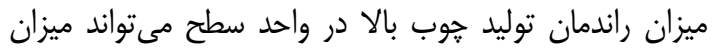

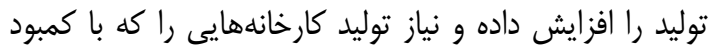

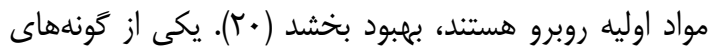

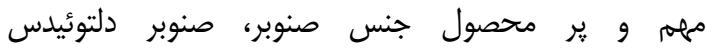
است كه در (Populus deltoides subsp. deltoides,)

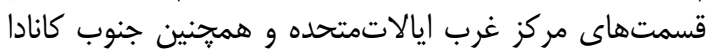

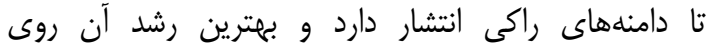

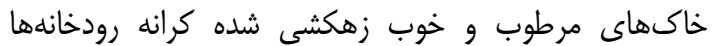

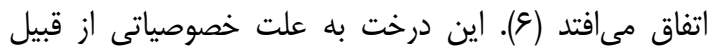
سهولت تجديد حيات، انطباق با شرايط مختلف رويشكاه، 
كيلومترى جنوب شهر سارى واقع شدهاند. جهت عمومى در

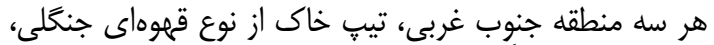

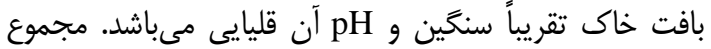

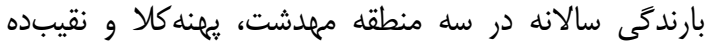

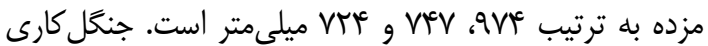

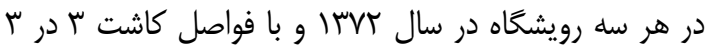

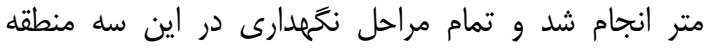

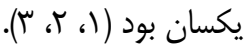

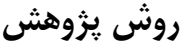

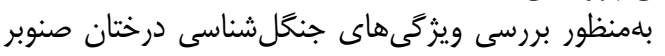

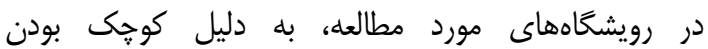

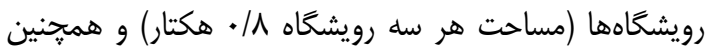

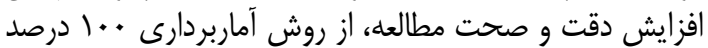

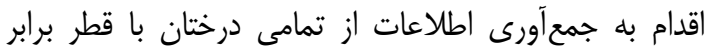

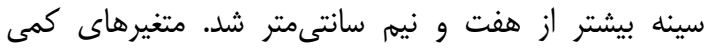

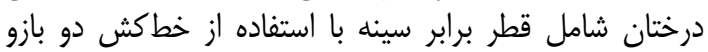

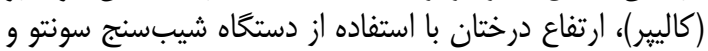

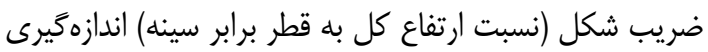

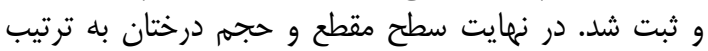
با استفاده از رابطه (ا) و (T) محاسبه شد.

$$
\begin{array}{ll}
G=\frac{\pi}{4} d^{2} & (\text { رابطه (I) رابطه () }
\end{array}
$$

در روابط فوق، G: سطح مقطع، d: قطر برابر سينه، H: ارتفاع

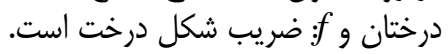

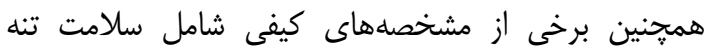

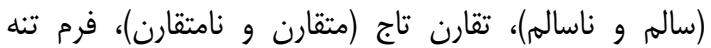

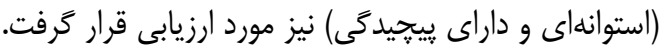
تجزيه و تحليل اطلاعات

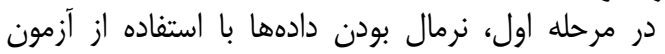

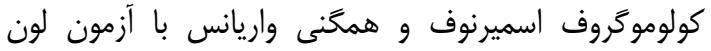

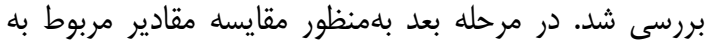

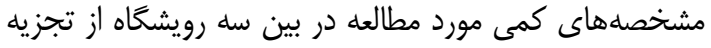

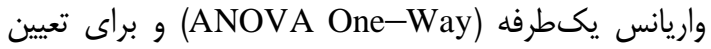

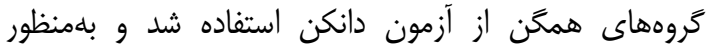

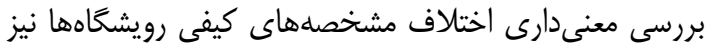
از آزمون كروسكال واليس استفاده شد. آناليز آمارى كليش آنيه دادهها با استفاده از نرمافزار

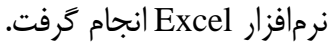

نتايج و بحث تركيب تونههاى درختى شناسايى شده

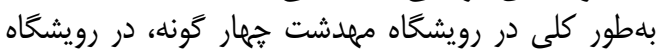

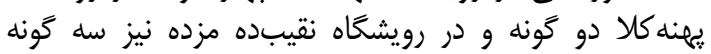
درختى بهجز گَونه صنوبر شناسايى شدند (جدول () ).
سطح دريا در وضعيت كمّى و كيفى توسكاى ييلاقى مؤثر بود.

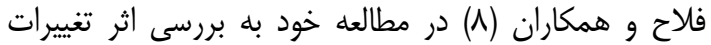

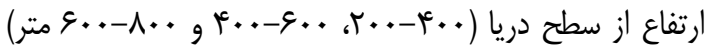

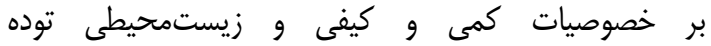

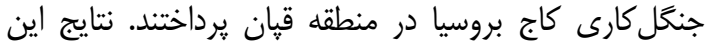

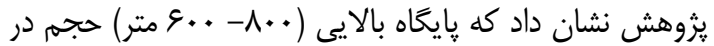

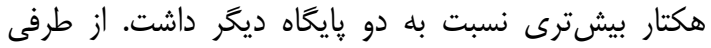

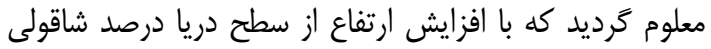

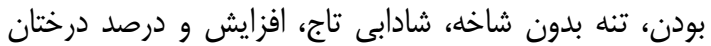

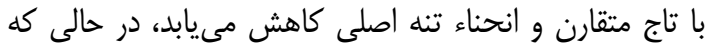

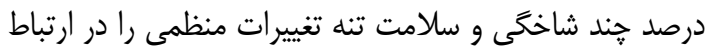

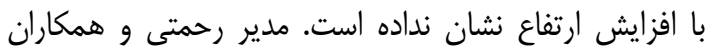

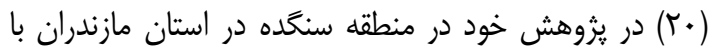

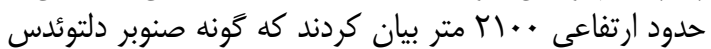

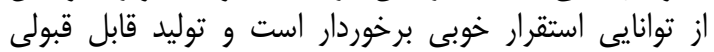

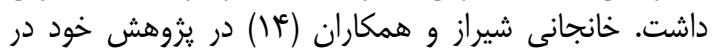

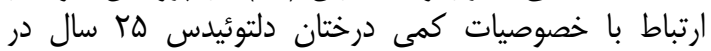

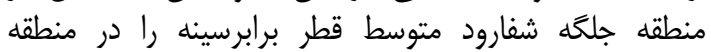

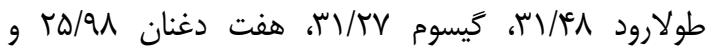

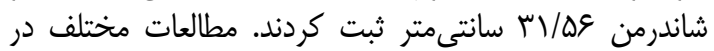

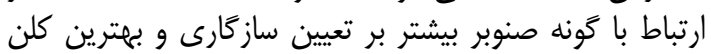

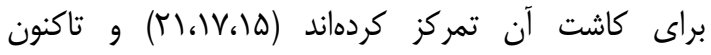

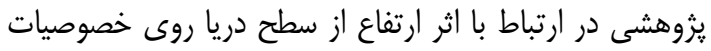

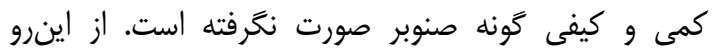

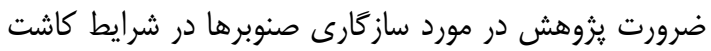

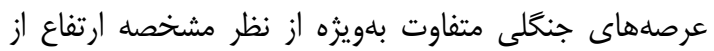

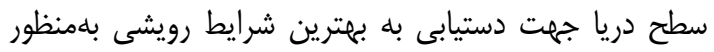

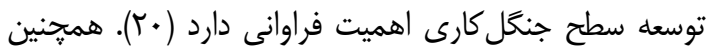

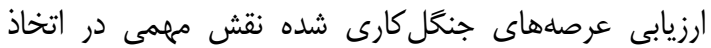

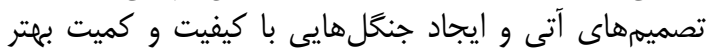

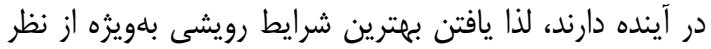

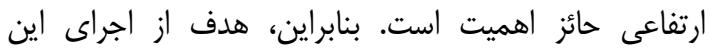

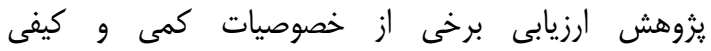
جنكل كارىهاى صنوبر دلتوئيدس در سه طبات طبقه ارتفاعى

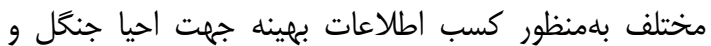
همجنين توليد جوب در نقاط مختلف ارتفاعى بود.

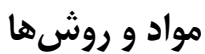 منطقه مورد بر روسى مورى}

بلمنظور بررسى اثر ارتفاع از سطح دريا روئ روى خصوصيات

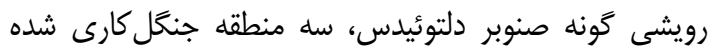

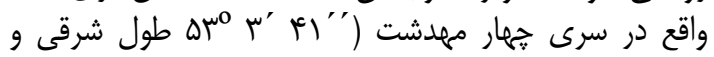
"19" متر)، سرى سه بهنه كلا ("

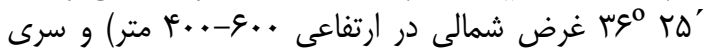

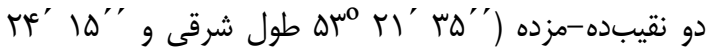

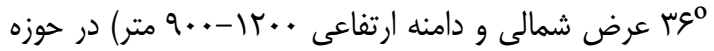

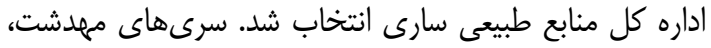
يهنه كلا و نقيبده مزده به ترتيب در فاصله 
Table 1. Percent of tree species composition in study sites

جدول (- درصد تر كيب گَونهاى درختى شناسايى شده در رويشعامهاى مورد مطالعه

\begin{tabular}{|c|c|c|c|}
\hline نقيبده مزده & يهنه كلا & مهدشت & كَونه درختى \\
\hline 94 & 99 & $M$ & صنوبر \\
\hline r & 1 & 1 & افرا \\
\hline 1 & r & 9 & انجيلى \\
\hline - & - & - & آزاد \\
\hline r & - & $\Delta$ & بلوط \\
\hline- & - & 1 & توسكا \\
\hline
\end{tabular}

با افزايش طبقه قطرى روند كاهشى در فراوانى درختان در هر

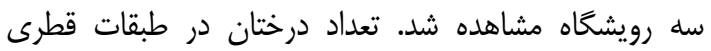

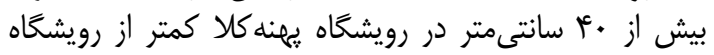

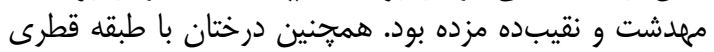

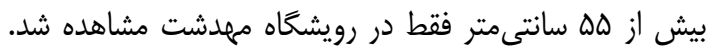

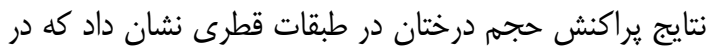

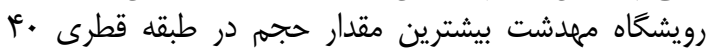

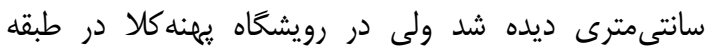

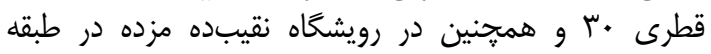
قطرى هץ ديده شد (شكل هم
مشخصات كمى درختان

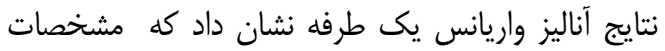

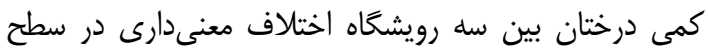

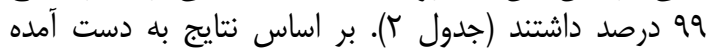

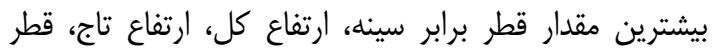

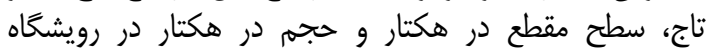

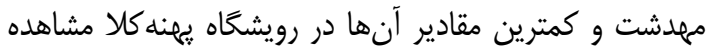

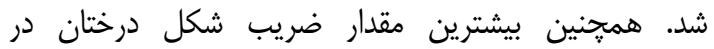

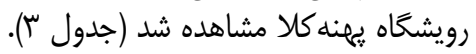

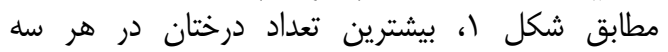

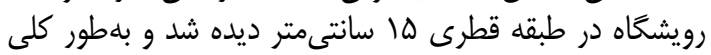

جدول r- نتايج آناليز واريانس يك طرفه مشخصات كمى درختان در رويشخاههاى مختلف Table 2. Results of One-Waye ANOVA for quantitative characteristic of trees in different sites

\begin{tabular}{|c|c|c|c|c|}
\hline F أماره F & ميانخين مربعات & درجه أزادى & منبع تغييرات & مشخصه كمى \\
\hline $1 T / V^{* *}$ & 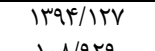 & $\begin{array}{c}r \\
c\end{array}$ & رويشكاه & قطر برابر سينه (سانتىمتر) \\
\hline & $\begin{array}{l}\text { l.N/qTq } \\
F M / F r / 1\end{array}$ & $\begin{array}{c}a t . \\
r\end{array}$ & خ خطاه & \\
\hline $1 \omega / 4 V^{* *}$ & TI/ & st. & خويساه & ارتفاع كل (متر) \\
\hline $\mid F / V r^{* *}$ & $\begin{array}{l}\Lambda I / I V \Delta \\
\Delta / \Delta \| 1\end{array}$ & $\begin{array}{c}r \\
s 4 .\end{array}$ & رويشاكاه & ارتفاع تاج (متر) \\
\hline $\mid N / V^{* *}$ & $\begin{array}{l}\Delta N / Q \& \Delta \\
\Gamma / \backslash Q 1\end{array}$ & $\begin{array}{c}r \\
\text { sq. }\end{array}$ & رويشاءه & قطر تاج (متر) \\
\hline $\mid f / \cdot e^{* *}$ & $\begin{array}{l}.1 . r \Delta \Delta \\
. . \cdot r\end{array}$ & $\begin{array}{c}r \\
\text { st. }\end{array}$ & خريشاء & سطح مقطع (مترمربع) \\
\hline $10 / 9 r^{* *}$ & $\begin{array}{l}\Delta \cdot / / D Q \\
\Gamma / \| F V\end{array}$ & $\begin{array}{c}r \\
s t .\end{array}$ & رويشاءه & حجم (مترمكعب) \\
\hline $\mathrm{V} / \mathrm{r \Lambda}^{* *}$ & $\begin{array}{l}. / T H A \\
. / . M F\end{array}$ & $\begin{array}{c}r \\
s 4 .\end{array}$ & رويشاكاه & ضريب شكل \\
\hline
\end{tabular}


جدول بـ- ميانكَين مشخصات كمى درختان (ميانكين ثلشتباه معيار) در رويشكاههاى مختلف Table 3. Mean of quantitative characteristic of trees (mean \pm SE) in different sites

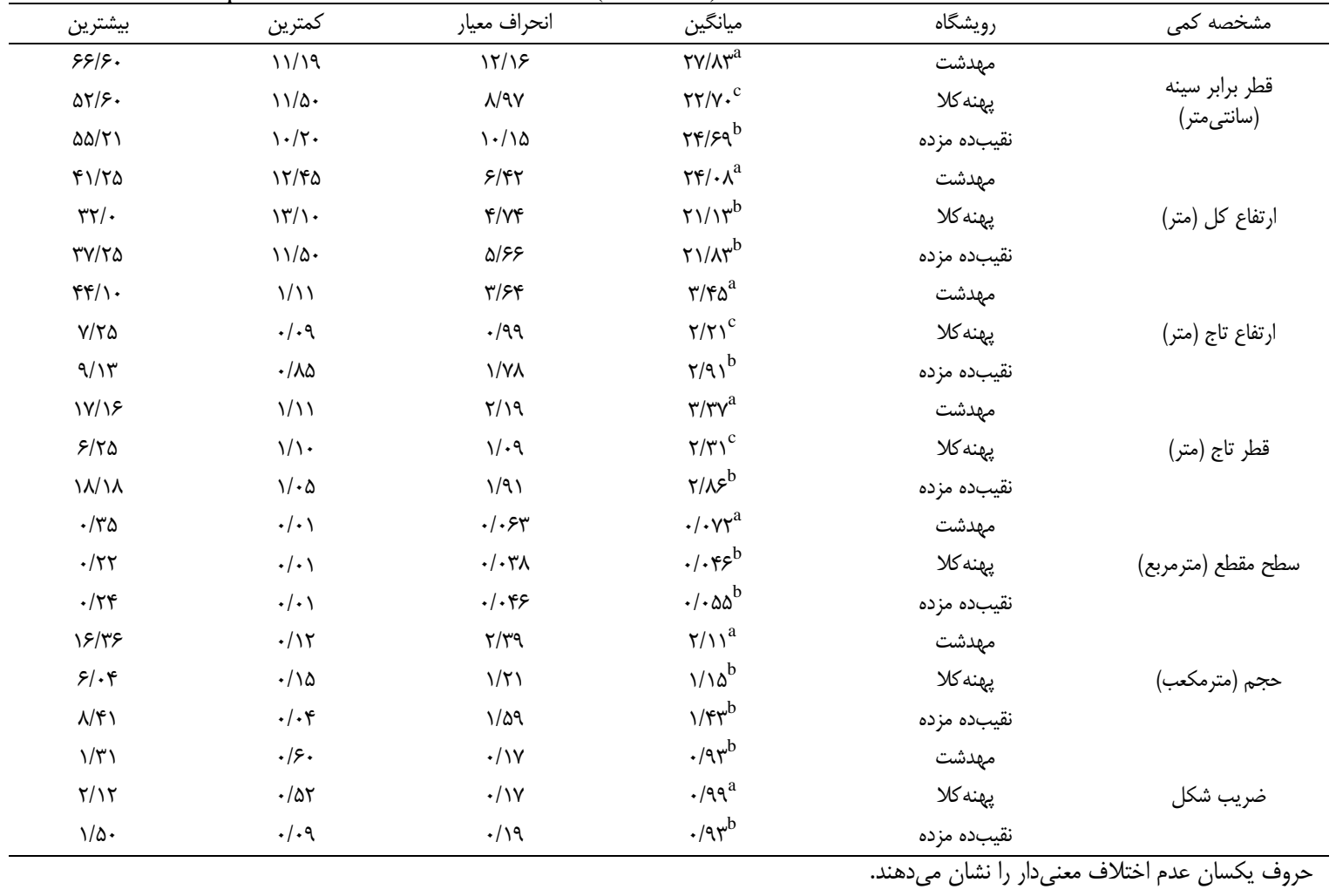

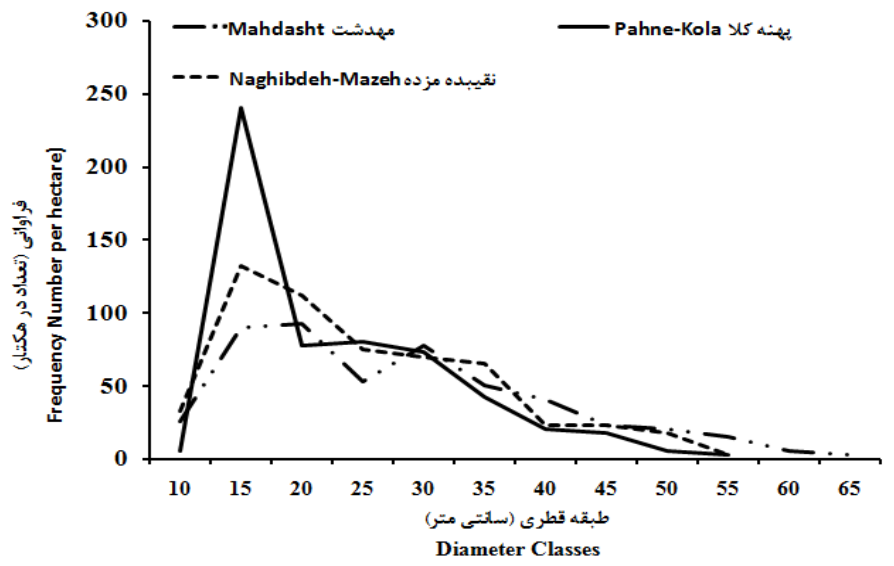

شكل 1- يراكنش تعداد در طبقات قطرى درختان در رويشكاههاى مختلف مورد مطالعه

Figure 1. Number of trees per hectare in diameter classes in study sites 


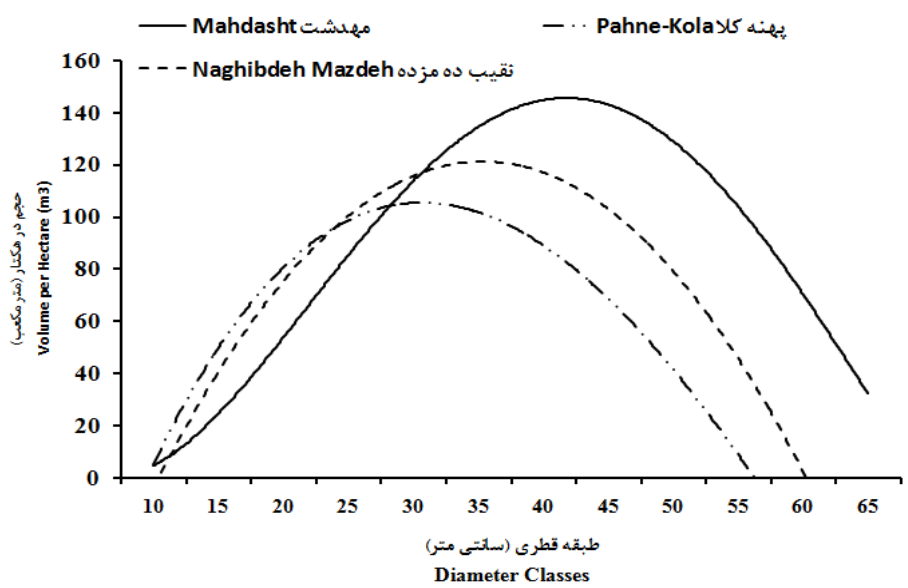

شكل r - يراكنش حجم در هكتار در طبقات قطرى درختان در رويشگاههاى مختلف مورد مطالعه

Figure 2. Volume of trees per hectare in diameter classes in study sites

تغييرات در تركيب گَونهها (جنَّلهاى طبيعى)، نرخ رشد،

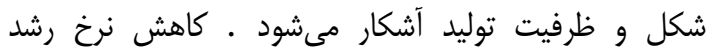

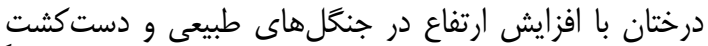

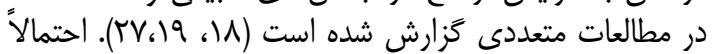

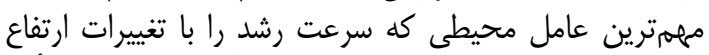

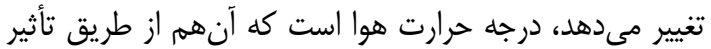

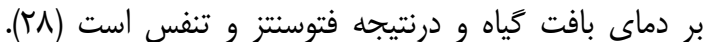

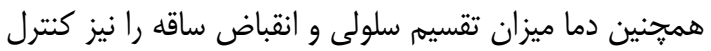

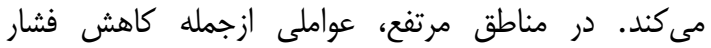

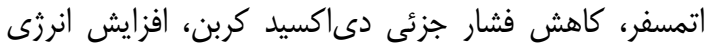

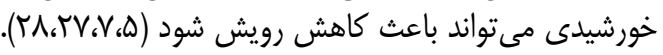

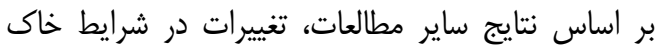

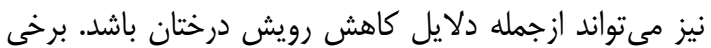

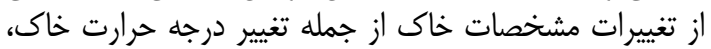

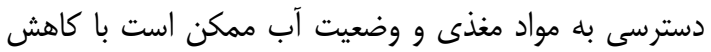

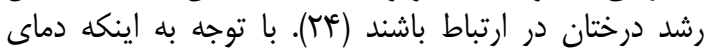

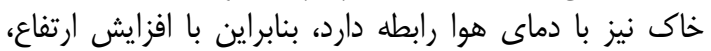

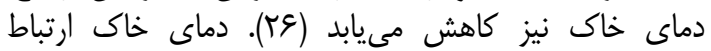

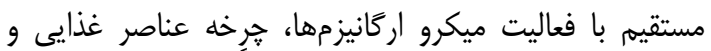

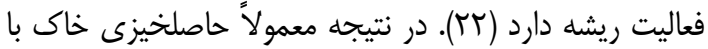

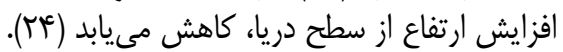

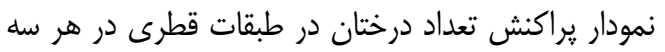

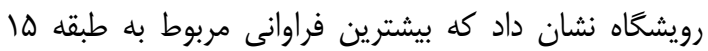

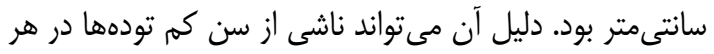

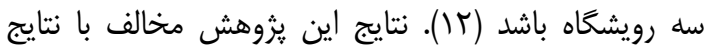

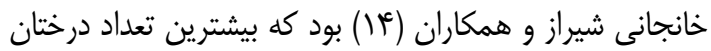

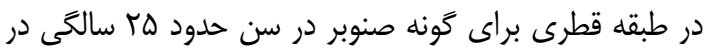

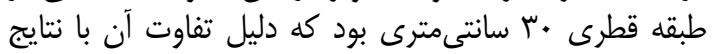

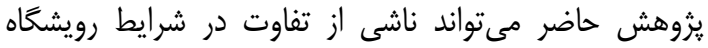

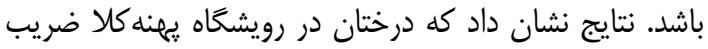

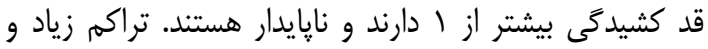

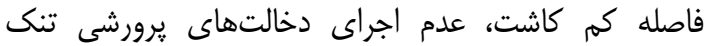

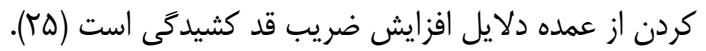

نتايج اين يزوهش نشان داد كه با افزايش ارتفاع از سطح

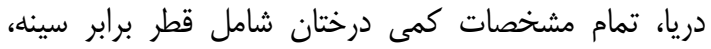

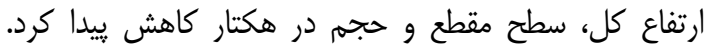

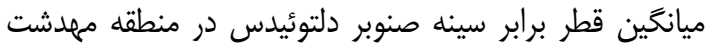
س مV/A

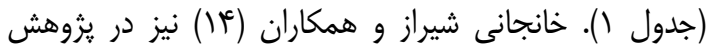

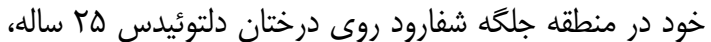

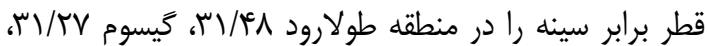

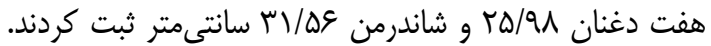

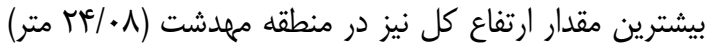

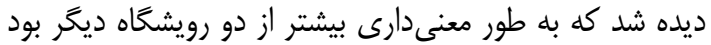

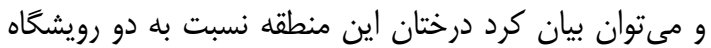

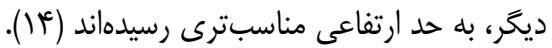

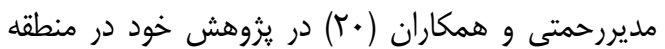

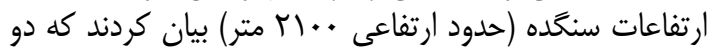

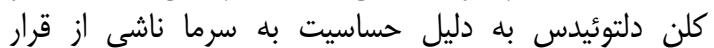

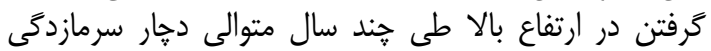

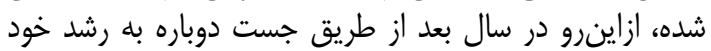

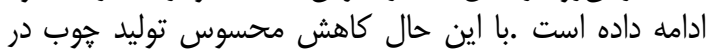

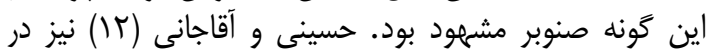

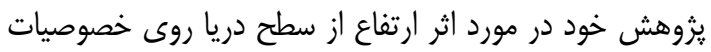

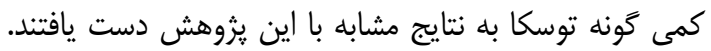

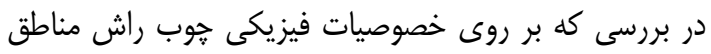

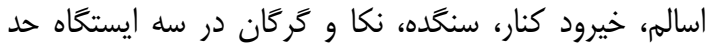

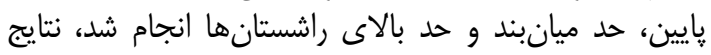

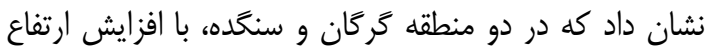

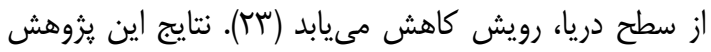

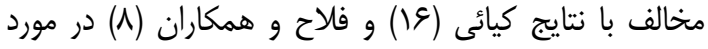

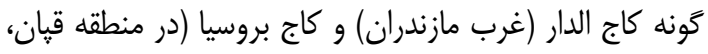

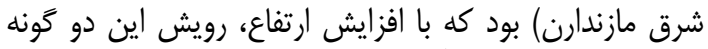

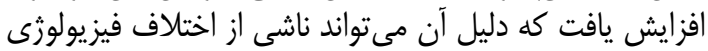
كونههاى مورد مطالعه باشد.

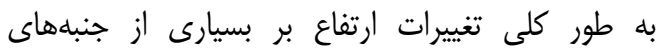

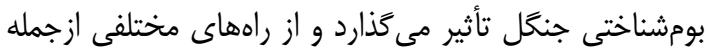


در رويشگاه يهنه كلا و بيشترين فراوانى درختان با تناه استوانهاى در رويشگًاه مهدشت مشاهده شدند (شكل بَ).
خصوصيات كيفى درختان در رويشكاههاى مورد مطالعه

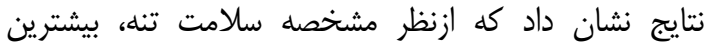

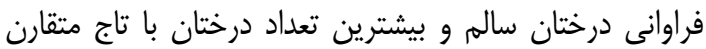

(a)

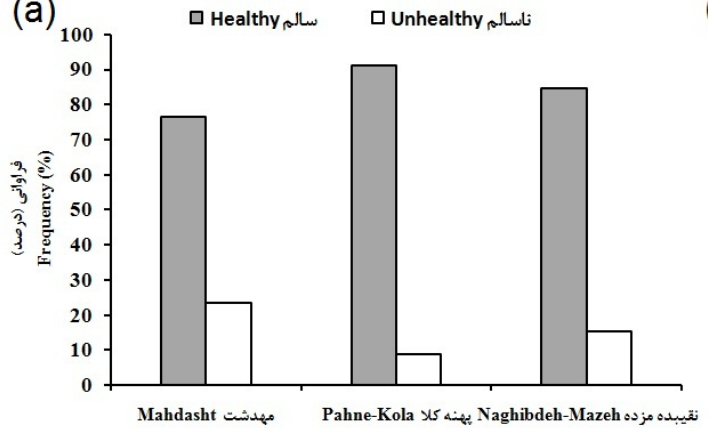

(b)

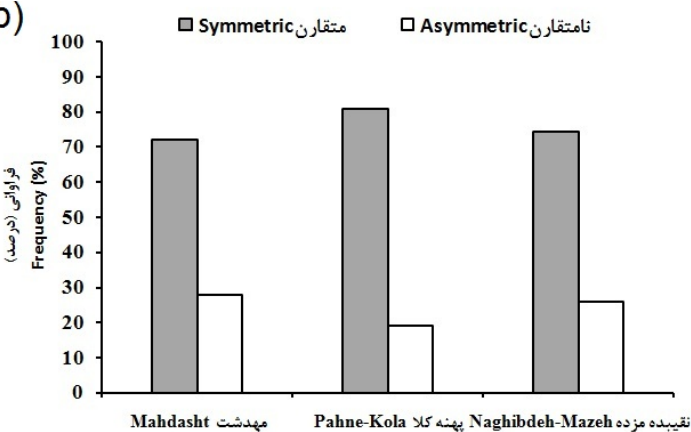

(c)

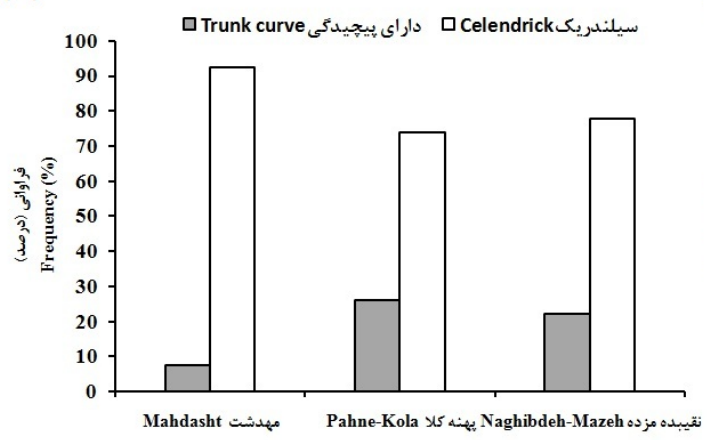

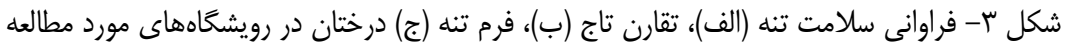

Figure 4. Frequency of trunk health (a), crown shape (b), trunk form (c) and trunk decay (d) in study sites

بيشترين مقدار امتياز سلامت تنه و تقارن تاج در رويشگاه

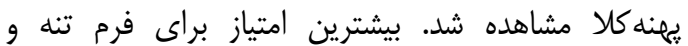

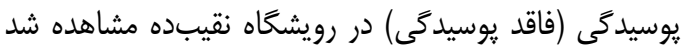

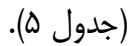

نتايج آزمون كروسكال واليس براى خصوصيات كيفى

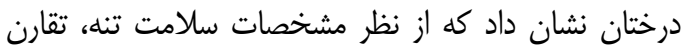

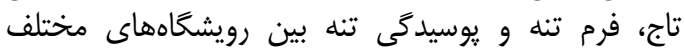

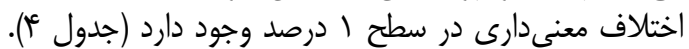

جدول ع- نتايج آناليز كروسكال - واليس مشخصات كيفى درختان در رويشخاههاى مورد مطالعه Table 4. Results of Kruskal walis analysis of Qualitative Characteristic of trees in study sites

\begin{tabular}{|c|c|c|c|c|}
\hline بررسى :وسيدگى تنه & 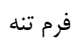 & تقارن تاج & سلامت تنه & \\
\hline $\mid 9 / \pi 1$ & $r \pi / \Lambda$. & f/Q & $18 / \mathrm{V}$. & مقدار كاى مربع \\
\hline r & r & r & r & درجه آزادى \\
\hline.$/ .1^{* *}$ & $.1 .1^{* *}$ & $.1 \cdot 1^{* *}$ & $.1 .1^{* *}$ & $P$ value \\
\hline
\end{tabular}


Table 5. Mean rank of Qualitative Characteristic of trees in study sites

$$
\text { جدول ه- متوسط امتياز مشخصات كيفى درختان در رويشگاههاى مورد مطالعه }
$$

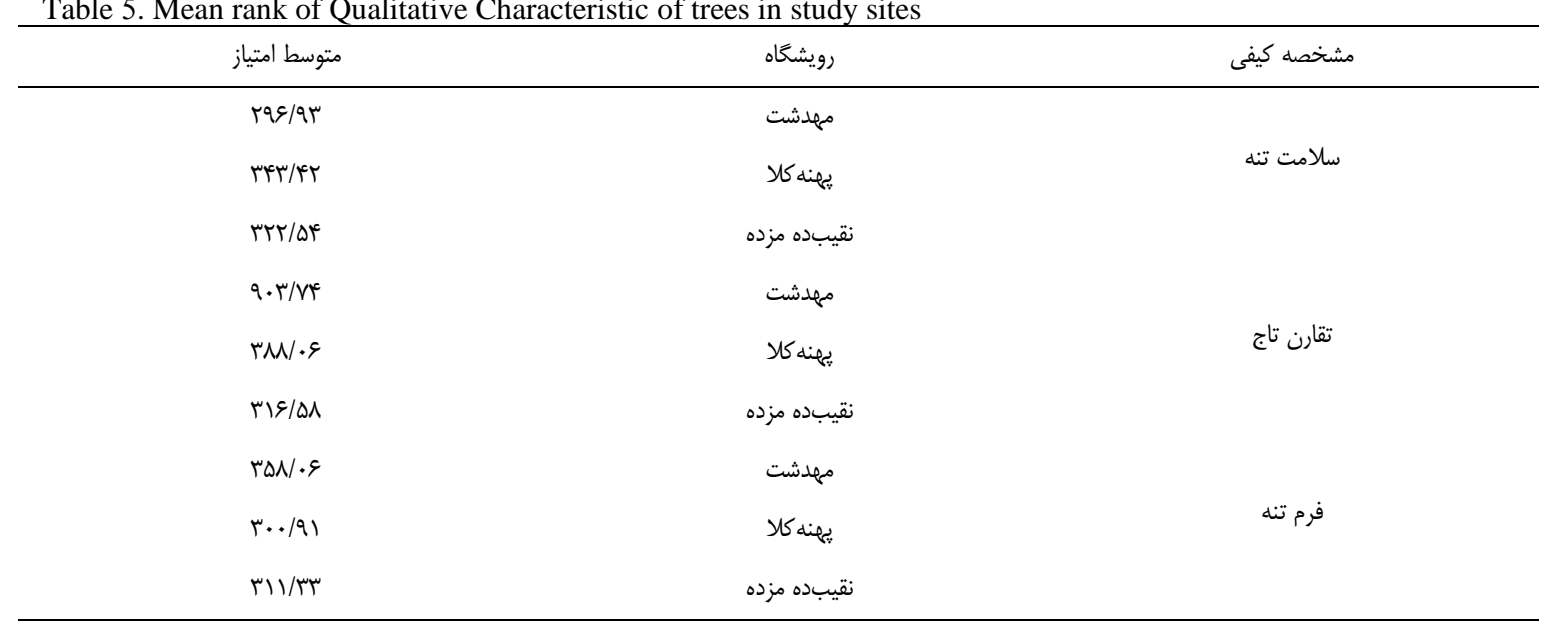

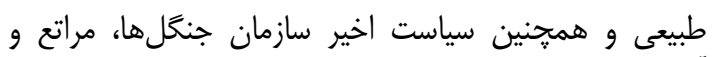

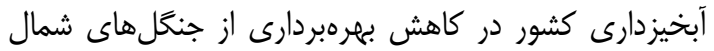

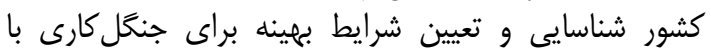

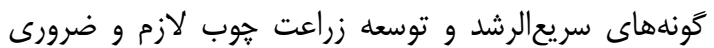

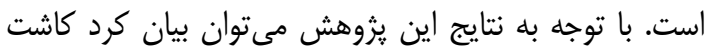

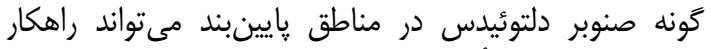

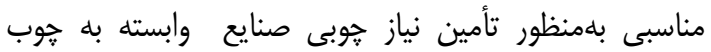

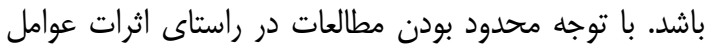

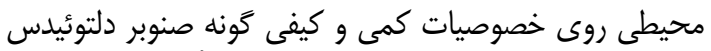

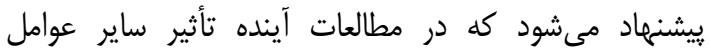

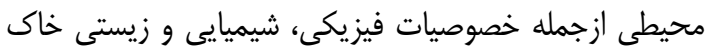

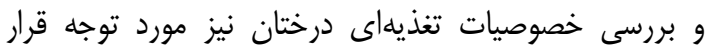

گيرد.

$$
\begin{aligned}
& \text { روند مشخص و تغييرات منظمى براى خصوصيات كيفى دئ دان }
\end{aligned}
$$

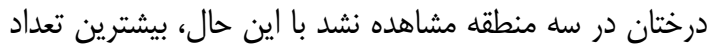

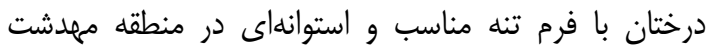

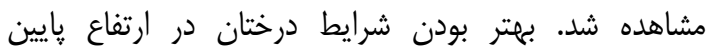

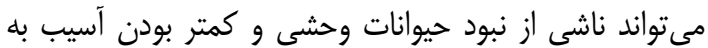

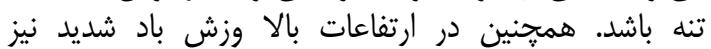

$$
\begin{aligned}
& \text { مىتواند باعث كاهش سلامت و كيفيت تنه درخت درنان شود }
\end{aligned}
$$

1. Anonymous. 2009. Forest Management Plan of Pahnekola. Published by Forests, Range and Watershed Management Organization of Iran, 930 pp (In Persian).

2. Anonymous. 2010. Forest Management Plan of Mahdasht. Published by Forests, Range and Watershed Management Organization of Iran, $860 \mathrm{pp}$ (In Persian).

3. Anonymous. 2011. Forest Management Plan of Mahdasht. Published by Forests, Range and Watershed Management Organization of Iran, $820 \mathrm{pp}$ (In Persian).

4. Bakhshipour, R., H. Ramezanpour and E. Lashkarboluki. 2012. Studying the effect of Pinus taeda and Populus sp. plantation on some forest soils properties (Case study: Fidareh of Lahidjan), Iranian Journal of Forest, 4(4): 321-332.

5. Benecke, U. and M.R. Davis. 1980. Mountain environments and subalpine tree growth. New Zealand Technical Paper Forest Research Institute. 70, 288 pp.

6. Cooper, D.T. and D.F. Van Haverbeke. 1990. Populus deltoides Bartr. ex Marsh. Eastern cottonwood: 530-536. In: Burns, R.M. and Honkala, B.H. (Eds.). Silvics of North America, Vol. 2: Hardwoods. Published by United States Department of Agriculture, Forest Service, USA, 654 pp.

7. Daubenmire, R.F. 1954. Alpine timberlines in the Americas and their interpretation. Butler University Botanical Studies, 119-136.

8. Fallah, A., Y. Kooch and A.A. Rastaghi. 2016. Effect of Altitude Changes on Quantitative and Qualitative Characteristics and Environmental Afforestation Stand of Pinus Brutia Ten, Journal of Environmental science and Technology, 18(2): 128-143.

9. Forotannejhad, M. 1969. Guidance of poplar plantation on lowlands in north of Iran. Ministry of Natural Resources of Iran, Tehran, 30 pp (In Persian).

10. Forouzesh-Sotgavaberi, R., M.T. Ahmadi, V. Etemad and H.R. Saeidi, 2009. Investigation on quantitative and qualitative characteristics of 19-years old plantation of Caucasian alder (Alnus subcordata) in Siahkal region, Iranian Journal of Forest, 1(2): 137.150. 
11. Gorji Bahri, Y., R. Faraji, S. kiadaliri, E. Abbassi and B. Gharib. 2009. The effect of thinning on growth and wood production of Caucasian alder (Alnus subcordata) plantation in Nowshahr region. Iranian Journal of Forest, 1(1): 43-55.

12. Hosseini, S. and H. Aghajani. 2017. An investigation on the quantitative and qualitative characteristics of Alnus subcordata with changing the elevation above sea level (Case study: Pahne Kolla district, Sari), Forest and Wood Products, 70(2): 293-301.

13. Isik, K. 1999. Altitudinal variation in Pinus brutia Ten: Seed and seedling characteristics. Silva Genetica. 35: 58-66.

14. Khanjani Shiraz, B., A. hemati, K. Pour tahmasy and H. Sardabi. 2014. Growth comparison of different poplar clones, planted on lowlands of west Guilan, Iranian Journal of Forest and Poplar Research, 21(3): 557-572.

15. Kiadaliry, SH., M. Tabari, F. Sarmadian and F. Ziai Ziabary, 2004. Effect of soil type on some quantitative and qualitative characteristics of Populus X. euramericana (Dode) Gunier. Pajouhesh and Sazandegi, 62: 45-50.

16. Kiaei, M. 2014. Investigation on wood properties of Eldar pine (Pinus eldarica Medw) and its relations to soil chemical and physical characteristics (in western of mazandarn province plantation), Iranian Journal of Wood and Paper Science Research, 29(2): 199-207.

17. Lashkar Bolouki, E., A. Modir-Rahmati, E. Kahneh and S.A. Mousavi koopar, 2010. Phenology and growth characteristics of seven clones of Populus deltoides in Astaneh Ashrafie, Guilan, Iranian Journal of Forest and Poplar Research, 18(4): 527-538.

18. Malcolm, D.C. 1970. Site factors and the growth of Sitka spruce. Unpub. PhD. Thesis, Edinburgh Univ.

19. Mayhead, G.J. 1973. The effect of altitude above sea level on the yield class of Sitka spruce. Scottish Forestry, 27: 231-237.

20. Modir-Rahmati, A.R., M. Calagari, R. Ghasemi and Hemmati A, 2015. Study of adaptability and growth of different cultivars of poplar and paulownia in mountainous altitudes of Northern Iran: a case study of Sang Deh rural district of Mazandaran, Iranian Journal of Plant Science, 28(2): 390-400.

21. Mousavi Koopar, S.A., A.R. Modir-Rahmati, E. Lashkar-Bolouki and E. Kahneh. 2011. Adaptation of Poplar clones in Safrabasteh, Guilan province, Iranian Journal of Forest and Poplar Research, 19(2): 326-339.

22. Nordmeyer, A.H. 1980. Tree nutrient concentrations near the treeline, Craigieburn Range, New Zealand. In: Benecke, U. and Davis, M.R. (Eds.). New Zealand Technical Paper Forest Research Institute, 70: 83-94.

23. Parsa Pajoh, D. 1976. The study of the physical quality of Iranian beech wood in different sites. Iranian journal of Natural resources, 34: 20-32.

24. Pearsall, W.H. 1950 Mountains and moorlands. New Naturalist, London.

25. Rouhi Moghaddam, A., E. Ebrahimi, S.M. Hosseini, A. Rahmani and M. Tabari. 2009. Comparison of growth characteristics of oak in pure and mixed plantations, Iranian Journal of Forest and Poplar Research, 17(2): 210-224.

26. Shanks, R.E. 1956. Altitudinal and microclimatic relationships of soil temperature under natural vegetation. Ecology, 37: 1-7.

27. Tranquillini, W. 1964. Photosynthesis and dry matter production of trees at high altitudes. In: The formation of wood in forest trees. Zimmermann M.H. (Ed.) pp: 505-518, Academic Press, New York.

28. Tranquillini, W. 1979. Physiological ecology of the alpine timberline-tree existence at high altitudes with special reference to the European Alps. Springer-Verlag, Berlin. 


\title{
Effect of Altitude on some Quantitative and Qualitative Characteristics of Populus Deltoids Trees
}

Masoud Naderi Varandi ${ }^{1}$, Ali Kialashaki ${ }^{2}$, Ramin Veisi ${ }^{3}$ and Ali Sheykheslami ${ }^{4}$

1- Ph.D. Student of Forestry, Chalous Branch, Islamic Azad University, Chalous, Iran

2- Associate Professor of Forestry, Noshahr Branch, Islamic Azad University, Noshahr, Iran. (Corresponding author: ali_kialashaki@iauns.ac.ir )

3- Associate Professor of Wood Industries, Chalous Branch, Islamic Azad University, Chalous, Iran

4- Assistant Professor of Forestry, Chalous Branch, Islamic Azad University, Chalous, Iran Received: April 7, 2018 Accepted: June 2, 2018

\begin{abstract}
In order to study the effect of altitude on some quantitative and qualitative characteristic of Populus deltoides in Mazandaran province, three sites were selected with elevations between 150 and $1200 \mathrm{~m}$ above sea level in Sari city. In each site, some tree traits such as diameter at breast height, total height, basal area, volume, form factor and qualitative variables including trunk health, crown shape and trunk form were measured and evaluated and quantitative and qualitative characteristics of trees in three sites were compared using one-way ANOVA and Kruskal Wallis tests. Results showed that the altitude had significant effects on qualitative and quantitative characteristics of Populus deltoides trees and trees in lower altitude ranges (150$300 \mathrm{~m})$ had a higher diameter and volume growth than trees in higher altitudes. According to results, the highest diameter $(27.83 \mathrm{~cm})$, height $(21.13 \mathrm{~m})$, basal area $\left(0.046 \mathrm{~m}^{2}\right)$, volume $(2.11$ $\mathrm{m}^{3}$ ) and the highest number of trees with cylindrical trunks were observed in Mahdasht. Tress in Pahnekola had the highest form factor (0.99) and with symmetrical crowns. According to results of this study, it can be concluded that planting with Populus deltoides trees in lower altitude ranges can increase the qualitative and quantitative characteristics of such trees and provides a suitable approach for supplying wood for wood-based industries.
\end{abstract}

Keywords: Silvicultural characteristics, Diameter and volume growth, Altitude 